\title{
Case report of gastric teratoma masquerading as neonatal intestinal obstruction
}

Rajib Chatterjee ${ }^{*} \mathbb{D}$, Abhijit Singh and Vivek Gharpure

\begin{abstract}
Background: Teratomas are rare in neonates. They are embryonic neoplasms containing elements from all the three germ layers. Commonly, they arise in the ovary and testis. Rarely do they arise from the gastrointestinal tract, as in our case. Intestinal obstruction in neonates is usually due to congenital obstructive malformation such as atresia and/or stenosis of the gastrointestinal tract with or without sepsis.

Case presentation: An outborn 5-day neonate, generally well before admission presented with non-bilious vomiting, abdominal distension and a palpable mass extending from the right to left flank across the centre. Bilious aspirates/vomiting usually indicates obstruction distal to the ampulla of Vater. Clear aspirates indicate obstruction proximal to the ampulla which usually present by second day of life.

Possibility of a mass lesion such as a "teratoma" causing gastrointestinal obstruction needs a high index of suspicion. Imaging studies of plain X-ray chest with abdomen enhanced our clinical suspicion which was promptly followed by ultrasonography of the abdomen and thereafter computerized tomography of the abdomen which provided corroborative evidence. Laparotomy on the seventh day of life confirmed the clinical suspicion and imaging evidence of an exogastric mass arising from the postero-inferior wall of the stomach along its greater curvature. Histopathology revealed a mature teratoma.

Conclusion: A high index of clinical suspicion by the paediatrician/neonatologist attending a neonate with abdominal distension suggestive of gastrointestinal obstruction, immediate cardiopulmonary, hemodynamic and circulatory stabilization, followed by prompt and appropriate imaging studies with timely surgical consultation holds the key to quality outcome.
\end{abstract}

Keywords: Alpha-fetoprotein, Mature teratoma, Neonate, Stomach

\section{Background}

Gastric teratomas are rare tumours in infants and children and constitute less than $1 \%$ of all teratomas occurring in children [1,2]. They are however the most common teratomas of the gastrointestinal tract, other sites being the tongue and oropharynx. Most of the cases occur in males whereas sacrococcygeal tumours occur commonly in females. Gastric teratomas may present as gastrointestinal bleeds or respiratory distress suggestive of intramural extensions. We report a 5-day boy

\footnotetext{
* Correspondence: drrajibchatterjees@yahoo.co.in

Department of Pediatrics, Rural Medical College, Pravara Institute of Medical Sciences, Loni, Ahmednagar, Maharashtra 413736, India
}

presenting with abdominal distension which on work up revealed a gastric teratoma.

\section{Case presentation}

A male neonate born of non-consanguineous marriage, weighing $3000 \mathrm{~g}$ delivered normally at term to a multigravida mother, was presented to us with abdominal distension on the fifth day of life with non-bilious vomiting and a history of poor feeding since the third day of life. Intestinal obstruction in neonates is usually due to congenital obstructive malformation such as atresia and/or stenosis of the gastrointestinal tract with or without sepsis. Bilious aspirates/vomiting usually indicates obstruction distal to the ampulla of Vater. Clear aspirates indicate obstruction proximal to the ampulla which 
usually present by the second day of life. Though he had passed meconium on the first and second days of life, he had constipation thereafter. Clinical examination revealed a weight of $2850 \mathrm{~g}$ and an altered general appearance, i.e. agitated cry, tachycardia and tachypnoea with shallow breathing, normothermic and dehydrated with prolonged capillary refill time (CRT). We also noted significant abdominal distension with shiny skin, guarding and inability to palpate abdominal viscera while awake. During sleep, a large, mobile mass extending from the right to the left flank across the midline was palpated. The mass was firm, non-tender and non-mobile with irregular surface, occupying the left hypochondrium and epigastric regions. Rectal exam indicated a normal faecal tone and stool in the rectal vault.

\section{Imaging tests}

Ultrasound abdomen revealed a solid-cystic mass with mixed echogenicity in left hypochondrium and continuous with gastric outline. Thoracic and abdominal X-ray (Fig. 1) revealed a gaseous bowel distension in the right flank and inferior abdomen, with opacity in the rest of the abdomen. Abdominal computerized tomography (CT) demonstrated a large, lobulated, heterogeneous, exogastric mass attached to the greater curvature of the

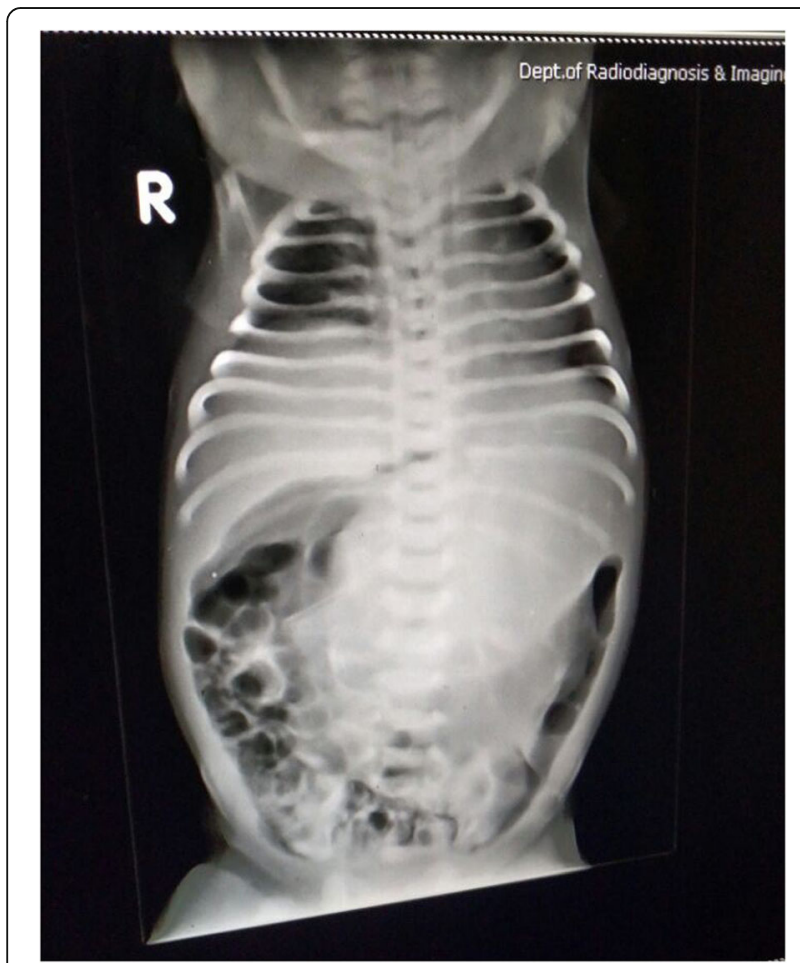

Fig. 1 Thoracic and abdominal $X$-ray revealed a gaseous bowel distension in the right flank and inferior abdomen, with opacity in the rest of the abdomen stomach displaying solid and cystic components with calcification and fat attenuation, suggestive of teratoma.

Laboratory tests revealed normoglycemia with a normal sepsis screen as well as normal arterial blood gases, renal function tests (RFT), liver function tests (LFT), and erythrocyte sediment rate.

Biochemical parameters and age-related serum alpha fetoprotein (AFP) levels were within normal limits.

\section{Management}

After stabilizing the neonate, exploratory laparotomy was performed on the seventh day of life which revealed a large solid-cystic mass $9.5 \mathrm{~cm} \times 5 \mathrm{~cm} \times 3 \mathrm{~cm}$ arising from postero-inferior wall of the stomach along its greater curvature (Fig. 2). The mass was excised and sent for histopathology. The defect was repaired and patient was shifted back to neonatal intensive care unit (NICU). Parenteral fluids were continued for $24 \mathrm{~h}$ then graduated nasogastric feeding for 2 days and thereafter full breastfeeds were supervised. During the postoperative period, haemoglobin monitoring revealed a fall to $10 \mathrm{~g} / \mathrm{dL}$ so transfusion was given on the 11th day of life. The child was discharged on the tenth postoperative day, i.e. 18th day of life.

Histopathological examination of the tumour revealed the presence of hair follicle, stratified squamous epithelium, mature glial tissue, adipose tissue, bone and cartilage suggestive of mature gastric teratoma.

\section{Discussion}

Gastric teratomas account for approximately $1 \%$ of the paediatric teratomas [1]. Teratoma in the sacrococcygeal region is the commonest tumour in neonates. Other common locations of teratomas in neonates and infants are gonads or the mediastinum [2]. Gastric teratomas usually occur in males and only less than a dozen female children with this tumour have been reported $[1,3,4]$. Preoperative diagnosis of gastric teratomas is difficult. Imaging studies demonstrate a mass with solid and cystic components. There may be internal calcifications. With contrast-enhanced CT scan, the tumour's origin and relation with adjacent viscera and blood vessels as well as the presence of metastasis can be confirmed. Diagnosis of teratoma rests on finding derivatives of all three germ layers on histology. When the tumours contain only two germ layer derivatives, the tumour is more appropriately termed as a dermoid. A gastric teratoma can be termed as mature or immature depending on the differentiation of the neuroglial tissue in it. When the tumour contains mature glial tissue along with other derivatives of the germinal layers, these can be considered as benign tumours. Malignant tumours contain immature neuroepithelial tissue, and the malignant components may be endodermal sinus tumour (EST), yolk sac 


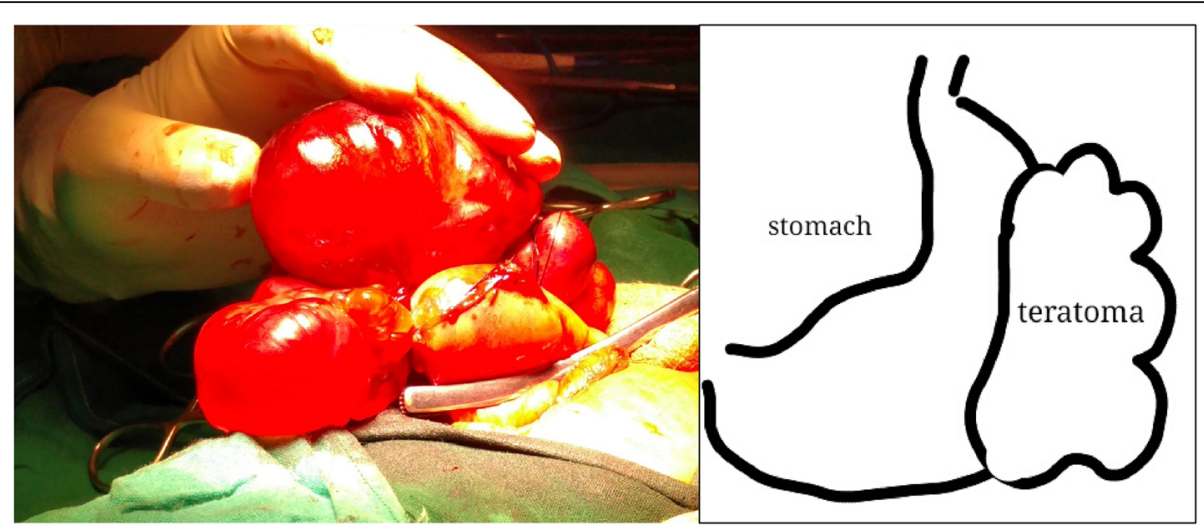

Fig. 2 After stabilizing the neonate, exploratory laparotomy was performed on the seventh day of life which revealed a large solid-cystic mass $9.5 \mathrm{~cm} \times 5 \mathrm{~cm} \times 3 \mathrm{~cm}$ arising from postero-inferior wall of the stomach along its greater curvature

tumour or choriocarcinoma. Patients with gastric teratomas, irrespective of histological features and invasion of adjacent organs have an excellent prognosis [5]. Complete surgical resection of the tumour is usually curative and sufficient; recurrence results from incomplete excision.

Our patient was the third issue of healthy nonconsanguineous parents (21-year-old mother, 25-yearold father). He has a 30-month-old sister and a 19month-old brother, both healthy. Patient was followed up for 6 months. Ultrasound of the abdomen was repeated for regrowth and serum AFP was done again to rule out recurrence but values were in normal range and the ultrasound was normal. The child has been doing well with normal level of age-related serum AFP at the 6-month follow-up. The parents were happy with their 6-month infant on follow-up. Regular follow-up of these patients with periodic serum AFP estimation is recommended to detect recurrence.

\section{Conclusion}

Neonatologists must maintain a high index of clinical suspicion for the various causes of gastrointestinal tract obstruction with the temporal age of presentation, from birth, in hours during the first week of life. Prompt follow-up of clinical suspicion by appropriate imaging and interpretation is the next step. Pursuing experienced paediatric surgical consultation for timely intervention is the key to good surgical outcome. Supportive care in NICU, both pre- and post-operative with appropriate fluids and antibiotics to cover for accompanying probable sepsis due to delayed referral from periphery, enabled successful discharge and follow-up.

\section{Abbreviations}

CRT: Capillary refill time; CT: Computerized tomography; RFT: Renal function tests; LFT: Liver function tests; AFP: Alpha fetoprotein; NICU: Neonatal Intensive Care Unit; EST: Endodermal sinus tumour

\section{Acknowledgements}

The authors would like to thank Dr D.K Ghosh, Prof of Pathology, RMC, PIMS(DU), Loni, for his expert opinion on histopathology slide.

\section{Authors' contributions}

RC diagnosed and supervised neonatal case management and was a major contributor in writing the manuscript. AS performed day-to-day monitoring of the case.VG performed surgical management of the case. All authors read and approved the final manuscript.

Funding

None

Availability of data and materials Not applicable

Ethics approval and consent to participate

Since the patient concerned is a newborn, written informed consent was obtained from the parent.

\section{Consent for publication}

Written informed consent was obtained from the parent for publication of this case report and accompanying images.

\section{Competing interests}

The authors declare that they have no competing interests.

Received: 1 October 2019 Accepted: 6 November 2019

Published online: 03 January 2020

References

1. Gengler JS, Ashcraft KW, Slattery P. Gastric teratoma: the sixth reported case in a female infant. J Pediatr Surg. 1995;30:889-90.

2. Gamanagatti S, Kandpal H. Gastric teratoma. Singapore Med J. 2007;48(4): e99-e101.

3. Gupta DK, Srinivas M, Dave S, Agarwala S, Bajpai M, et al. Gastric teratoma in children. Pediatr Surg Int. 2000;16:329-32.

4. Skinner MA, Plumley DA, Grosfeld JL, Rescorla FJ, West KW, et al. Gastrointestinal tumors in children: an analysis of 39 cases. Ann Surg Oncol. 1994;1:283-9.

5. Ijaz L, Aslam I, Sheikh A, Mirza B. Mature gastric teratoma: the mixed exogastric and endogastric variety. APSP J Case Rep. 2011;2:17.

\section{Publisher's Note}

Springer Nature remains neutral with regard to jurisdictional claims in published maps and institutional affiliations. 